Research Paper

\title{
The prognostic value and potential mechanism of Matrix Metalloproteinases among Prostate Cancer
}

\author{
Xinyu Geng\#, Chunyang Chen\#, Yuhua Huang, Jianquan Hou ${ }^{凶}$ \\ Department of Urology, The First Affiliated Hospital of Soochow University, 215006, Suzhou, Jiangsu, China. \\ \#These authors contribute equally to the current work. \\ $\triangle$ Corresponding author: Jianquan Hou, MD, PhD, E-mail: houjianquan@suda.edu.cn, Department of Urology, The First Affiliated Hospital of Soochow \\ University, 215006, Suzhou, Jiangsu, China. \\ (c) The author(s). This is an open access article distributed under the terms of the Creative Commons Attribution License (https://creativecommons.org/licenses/by/4.0/). \\ See http://ivyspring.com/terms for full terms and conditions.
}

Received: 2020.04.07; Accepted: 2020.06.03; Published: 2020.06.21

\begin{abstract}
Background: Matrix Metalloproteinases (MMPs) play an indispensable role in the initial alteration and development of $\mathrm{PCa}$. We tried to generate an MMP-related prognostic signature (MMPS) in prostate cancer (PCa).

Methods: TCGA-PRAD, MSKCC/GSE21032, GSE1 16918, GSE70769 cohorts were enrolled to assess the prognostic value of MMPs. The least absolute shrinkage and selection operator (LASSO) Cox regression was employed to generate the MMPS signature. The log-rank test and Kaplan-Meier (K-M) survival curve were applied to show the difference RFS, The receiver operating characteristic (ROC) curve and area under the ROC curve (AUC) was plotted to predict the accuracy of signature. CIBERSORT was conducted to analyze the different immune infiltration in MMPS-H and MMPS-L groups. Potential signaling pathways activated in the MMPS-H groups by Metascape.

Results: MMP1, MMP7, MMP11, MMP24 and MMP26 were selected by LASSO regression and established the MMPS predict signature. The MMPS showed the high prognostic value in TCGA-PRAD training cohort (AUC=0.714) and validation cohorts (GSE1 16918: AUC=0.976, GSE70769: AUC=0.738, MSKCC: $A \cup C=0.793$ ). Pid integrin 1 pathway, G2M checkpoint, and response to growth factor signaling pathways were activated in MMPS-H group, patients with the high MMPS risk score and low M2 macrophage showed the worst recurrence-free survival (RFS).

Conclusion: MMPs involved and played an essential role in the tumorigenesis and biochemical recurrence in PCa patients. The MMPS signature could accurately predict the recurrence of PCa patients and validated in several cohorts.
\end{abstract}

Key words: prostate cancer, matrix metalloproteinases, prognostic signature, LASSO regressio

\section{Introduction}

Prostate cancer (PCa) caused a massive health burden for males, especially older males, around the whole world. The cancer-specific mortality of PCa is high to $6.6 \%$, there are more than three hundred thousand patients die from PCa each year [1-3]. Methods to inhibit the function of androgen receptor are sufficient to protect the PCa patients; however, the effeteness could only insist for about two years, and finally step into the advanced castration-resistant $\mathrm{PCa}$ (CRPC) [4-7]. Therefore, it is necessary to find the prognostic signature and potential treatment targets to protect the patients from the harm of the advanced
PCa stage.

Matrix Metalloproteinases (MMPs) are zincdependent proteases that display a specific proteolytic activity against a broad range of substrates located on the extracellular matrix (ECM). There are about 23 MMPs were reported in human beings to regulate the cell functions through growth factors, cell-surface receptors, cytokines, chemokines and proteases [8, 9]. The typical structure of MMPs consists of an Nterminal zymogenic propeptide domain ( $\sim 80$ amino acids), a metal-dependent catalytic domain ( 170 amino acids), a linker region ( 15-65 amino acids), 
and a C-terminal hemopexin-like domain ( 200 amino acids) $[10,11]$. The relevant physiological function of MMPs, as well as the increased or decreased expression of them could affect the development of diabetes, neurological disorders, chronic inflammatory disease and cancers [12-16]. The MMPs are divide in different based on the function, MMP1, MMP8 and MMP13 belong to collagenases, MMP7 and MMP26 are matrilysins, MMP3, MMP10 and MMP11 are stromelysins, MMP2 and MMP9 are gelatinases, MMP14-17, MMP24 and MMP25 are in the membrane-type group, MMP 12 is metalloelastase, MMP20 is enamelysin, while the other MMPs are not in any specific subgroup [17].

In the past decades, several pieces of research demonstrated the function of MMPs in cancer. Qi et al. [18] reported that the CCL7 chemokine could regulate the MMP-9 mediated collagen degradation to promote the invasion and metastasis of liver cancer. Furuya et al. [19] illustrated a urine-based protein-determined signature to predict the bladder diagnostic, of which including MMP9 and MMP10. Singh et al.[20] revealed a 15 gene signature, identified by macrophages-tumor interactions, to predict the outcome of breast cancer, of which including MMP1 and MMP9. Therefore, we consider building a MMPs related prognostic signature in PCa patients.

\section{Methods}

\section{Data acquirement from enrolled cohorts}

In the current study, we concerned the TCGA-PRAD cohort with the RNA sequence data from the platform based on the Illumina system and clinical characteristics download from UCSC (https://gdc.xenahubs.net/download/TCGA-PRAD. htseq_fpkm.tsv.gz), We transferred the data from fragments per kilobase of non-overlapped exon per million fragments (FPKM) to into transcripts per kilobase million (TPM) values. Those mRNAs with TPM less than 1 in over $90 \%$ samples were considered noise and removed from downstream analysis. There are 52 normal samples and 500 tumor samples from 496 patients in TCGA-PRAD cohort. Meanwhile, sequence data of three cohorts from the Gene Expression Omnibus 36 (GEO, http:/ / www.ncbi.nlm. nih.gov/geo/) was also obtained, including MSKCC/ GSE21032, GSE116918, GSE70769 cohorts. The details of all the four cohorts are listed in Table 1 . The genetic alterations of MMPs in patients with $\mathrm{PCa}$ were illustrated via the cBioPortal platform (http:/ /www. cbioportal.org/) [21, 22], which recorded the missense and truncating mutations as well as amplification and deep deletion. The correlation between DNA methylation and mRNA expression of each MMP was also tested with cBioPortal.

Table 1. The information of training and validation cohorts

\begin{tabular}{|c|c|c|c|c|}
\hline Cohorts & Total & Non-BCR (n) & $\mathrm{BCR}(\mathrm{n})$ & Download Link \\
\hline $\begin{array}{l}\text { TCGA- } \\
\text { PRAD }\end{array}$ & 496 & 426 & 90 & $\frac{\text { https://gdc.xenahubs.net/download }}{\text { LTCGA-PRAD.htseq_fpkm.tsv.gz }}$ \\
\hline $\begin{array}{l}\text { MSKCC / } \\
\text { GSE21032 }\end{array}$ & 140 & 105 & 35 & $\begin{array}{l}\text { http://cbio.mskcc.org/cancergenomi } \\
\text { cs/prostate/data/ }\end{array}$ \\
\hline GSE116918 & 248 & 193 & 55 & $\begin{array}{l}\text { https://www.ncbi.nlm.nih.gov/geo/ } \\
\text { query/acc.cgi?acc=GSE116918 }\end{array}$ \\
\hline GSE70769 & 92 & 48 & 44 & $\begin{array}{l}\text { https://www.ncbi.nlm.nih.gov/geo/ } \\
\text { query/acc.cgi?acc=GSE70769 }\end{array}$ \\
\hline
\end{tabular}

\section{Establishment and validation of MMP-related prognostic signature}

The least absolute shrinkage and selection operator (LASSO) Cox regression models were constructed using the package "glmnet". By utilizing all the concerned genes, the function returns a series of values of $\lambda$ and models. The coefficients of the majority of the original genes were penalized to zero in line with the increasing values of the tuning parameter $\lambda$. The $\lambda$ was chosen when the partial likelihood deviance reached its lowest. A suitable model was chosen based on the 10-fold cross-validation of the function cv.glmnet. Using the function lambda.min, the remaining genes with non-zero LASSO coefficients were obtained. The risk score for each patient with LAC was calculated using the linear combination of each TPM of the gene multiplied by the LASSO coefficient of each gene. For the validation in three GEO cohorts, the risk score of each patient was also calculated by the MMP-related prognostic signature.

The log-rank test and Kaplan-Meier (K-M) survival curve were employed to show the difference RFS in both signal gene expression and risk score polarized high and low groups, while Cox regression conducted to generate the hazard ratio (HR) from high to low groups, with the use of "survival" and "survminer" packages. The receiver operating characteristic (ROC) curve and area under the ROC curve (AUC) was plotted to predict the accuracy of the signature.

\section{Different expression genes (DEGs) identification and pathway enrichment}

The DEGs analysis was performed with $R$ package "limma", and the fold-change $>0.5$ and P-value $<0.05$ were used as criteria to screen for DEGs between the MMP- related high and low risk groups. We subsequently performed a functional enrichment analysis to find the potential signaling pathways activated in the high risk groups by Metascape (http://metascape.org) [23]. 

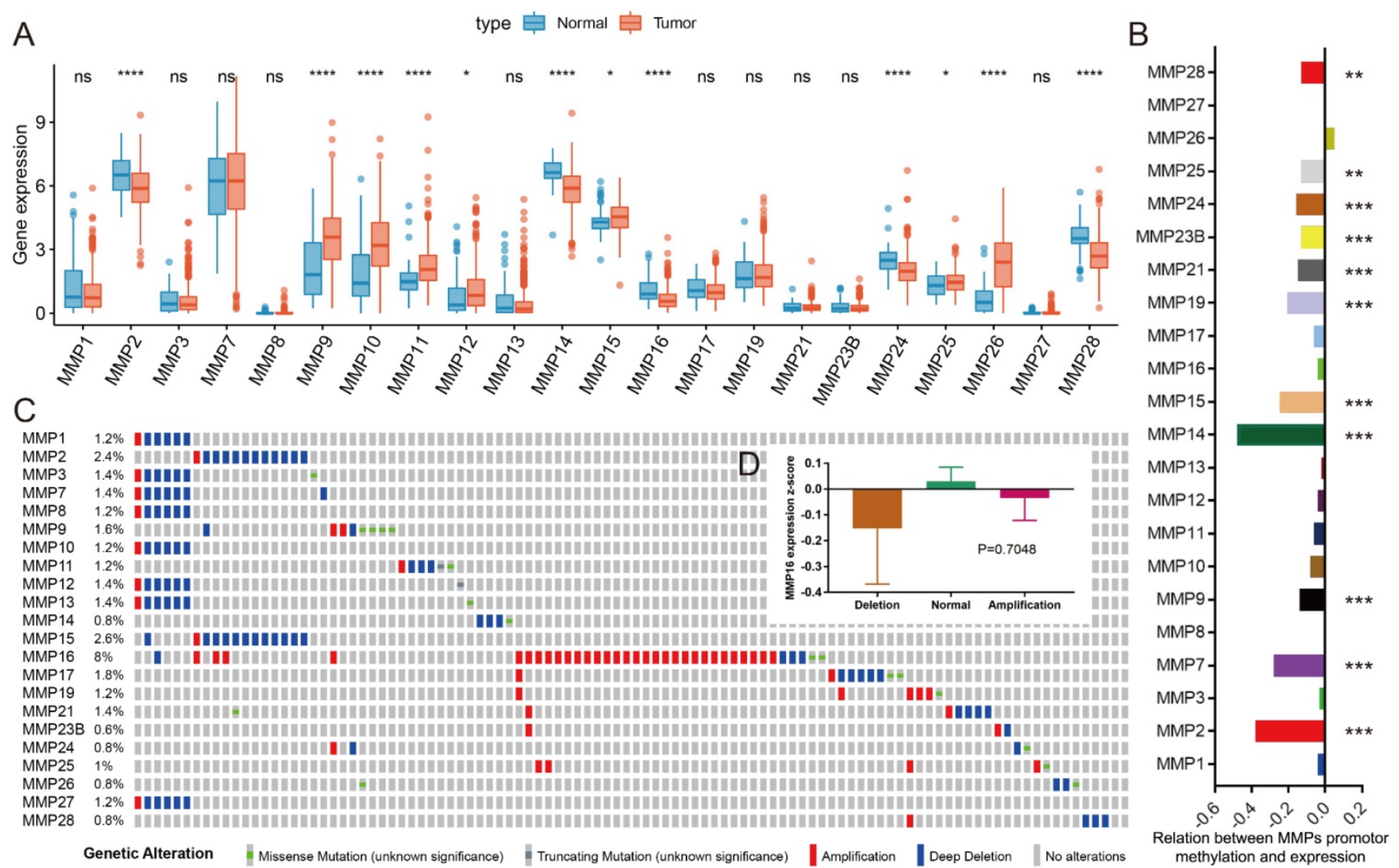

Figure 1. Overview of MMPs in PCa. (A) The mRNA expression of 22 MMPs among PCa tissues and normal prostate tissues; (B) The correlation of DNA methylation and mRNA expression of 22 MMPs; (C) The genetic alteration of 22 MMPs in PCa patients; (D) The amplification and deletion don't affect the mRNA expression of MMP16.

\section{Immune infiltration and prognostic value}

To furthermore analysis how immune cell affects the PCa patients in the high and low MMP-related risk groups, as well as the combined prognostic value, we use the TPM data to generate the results of the infiltration rate of 22 immunocytes with CIBERSORT (https://cibersort.stanford.edu/) [24], in the estimated analysis, the Mixture file was made with gene symbol and sequencing values, one thousand was set as permutation value for statistical analysis, and disable quantile normalization was selected. What's more, the association between gene expression and immune infiltration was also analyzed by a website called tumor immune estimation resource (TIMER) (https://cistrome.shinyapps.io/timer) served for analysis of tumor-infiltrating immune cells [25].

\section{Statistical methods}

The Student's test was used for statistical comparison among two subtypes. The ANOVA test was operated for analysis of more than two groups. Pearson correlation coefficient test was employed to assess the relationship between two factors, coexpression of two genes, or with the risk score. The statistical analysis of this research was implemented through R language (https://www.r-project.org/). P-value $<0.05$ was considered to be statistically significant.

\section{Results}

\section{A comprehensive view of MMPs in PCa}

After the search of the literature and extract the available data from TCGA-PRAD, the expression of 22 MMPs were listed in Figure 1A comparing between normal and tumor samples. We could see that about half MMPs are highly expressed in tumor tissues as compared with normal tissues, including MMP9, MMP10, MMP11, MMP12, MMP15, MMP25, MMP26 (all, $\mathrm{P}<0.05$ ), while some other MMPs decreased in tumor tissues, including MMP2, MMP14, MMP16, MMP24, MMP28(all, $\quad \mathrm{P}<0.05)$. DNA methylation is one of the risk factors that impact the mRNA level of genes, we analyzed the association between DNA methylation and mRNA expression for MMPs, and found that the DNA methylation level of MMP2, MMP7, MMP9, MMP14, MMP15, MMP19, MMP21, MMP23B, MMP24, MMP25 and MMP28 could impact its mRNA level in PCa (all, $\mathrm{P}<0.05$ ) (Figure 1B). Genetic alteration is another risk factor that affects mRNA expression. We found that the rate of genetic change in most MMPs was shallow in 
TCGA-PRAD patients, while the genetic alteration rate of MMP16 is high to 2.6\% (Figure 1C), however, when we compared the mRNA expression of MMP16 in different genetic alteration subgroups, there was no difference, of which means that the genetic alteration of MMP16 does no effect to the mRNA expression (Figure 1D).

\section{Establishment of the MMP-related prognostic signature}

We conducted the LASSO regression analysis to generate the signature, MMP8, MMP21, MMP23B and MMP27 were excluded by the low mRNA level, then 5 RFS associated MMPs were extracted to contract the prognostic signature, including MMP1, MMP7, MMP11, MMP24 and MMP26 (Figure 2). Then we reviewed the expression and prognostic value of these 5 MMPs. As shown in Figure 3A, MMP11 and MMP24 is the risk factors for unfavorable RFS (MMP11: $\mathrm{HR}=3.12, \quad 95 \% \quad \mathrm{CI}=1.91-5.09, \quad \mathrm{P}<0.001 ; \quad \mathrm{MMP} 24$ : $\mathrm{HR}=1.87,95 \% \mathrm{CI}=1.15-3.06, \mathrm{P}=0.007)$, while $\mathrm{MMP} 1$ $(\mathrm{HR}=0.59, \quad 95 \% \quad \mathrm{CI}=0.37-0.95, \quad \mathrm{P}=0.034), \quad \mathrm{MMP7}$ $(\mathrm{HR}=0.73,95 \% \mathrm{CI}=0.46-1.17, \mathrm{P}=0.186)$ and MMP26 $(\mathrm{HR}=0.40,95 \% \mathrm{CI}=0.25-0.64, \mathrm{P}<0.001)$ are the protect factors for $\mathrm{PCa}$ patients and associated with the prolonged RFS Subsequently, we analyzed the correlation of the five MMPs expression and the expression form in different clinicopathological subgroups. MMP7 expression is highly associated with MMP1, MMP11 and MMP24, as well as MMP11 and MMP24, while MMP11 expression is negatively associated with MMP26 (Figure 3B, all, $\mathrm{P}<0.05$ ). As to the expression in different Gleason score, MMP11 and
MMP24 mRNA level is higher in advanced Gleason score $\geq 8$ group, while MMP26 is higher in Gleason score $\leq 7$ group (Figure $3 \mathrm{C}$, all, $\mathrm{P}<0.05$ ). To pathology $\mathrm{T}$ stage subgroups, patients with the advanced $\geq \mathrm{T} 3$ stage shown a higher MMP11 and lower MMP26 (Figure 3D, all, $\mathrm{P}<0.05$ ).

For each MMP, the LASSO analysis generated a coefficient, the risk score formula of the signature is: MMPS risk score $=-0.04703^{*} \mathrm{MMP} 1_{\exp }-0.0223^{*}$ $\mathrm{MMP7}_{\text {exp }}+0.336547^{*} \mathrm{MMP} 11_{\exp }+0.218207^{*} \mathrm{MMP} 4_{\exp }$ $-0.11865^{*}$ MMP26 exp. The risk score of each patient in TCGA-PRAD cohort, the outcome status of each patient and the expression of the enrolled five MMPs were displayed in Figure 4A. Patients with high risk, whose risk score higher than the median value of all the patients, are defined as the MMPS-H group, and the others are in MMPS-L group. Patients in MMPS-H group shown a poor prognosis and shorter RFS, as comparing with MMPS-L group (Figure 4B, $\mathrm{HR}=3.43$, $95 \% \mathrm{CI}=2-5.86, \mathrm{P}<0.001)$. The predicted accuracy of the MMP-related signature is evaluated by the ROC curve, shown a good result with the 1-year (AUC $=0.714), \quad 3$-year $\quad(\mathrm{AUC}=0.735)$, and 5-year $(A U C=0.679)$ RFS (Figure 4C).

\section{Validation of the MMP-related prognostic signature}

To confirm the prognostic value of the MMPrelated signature in PCa patients. A total of $480 \mathrm{PCa}$ patients from GSE116918, GSE70769 and MSKCC cohorts. The risk score of the patients was calculated with the above-mentioned formula. In GSE116918 cohort, patients in the MMPS-H group shown a poor
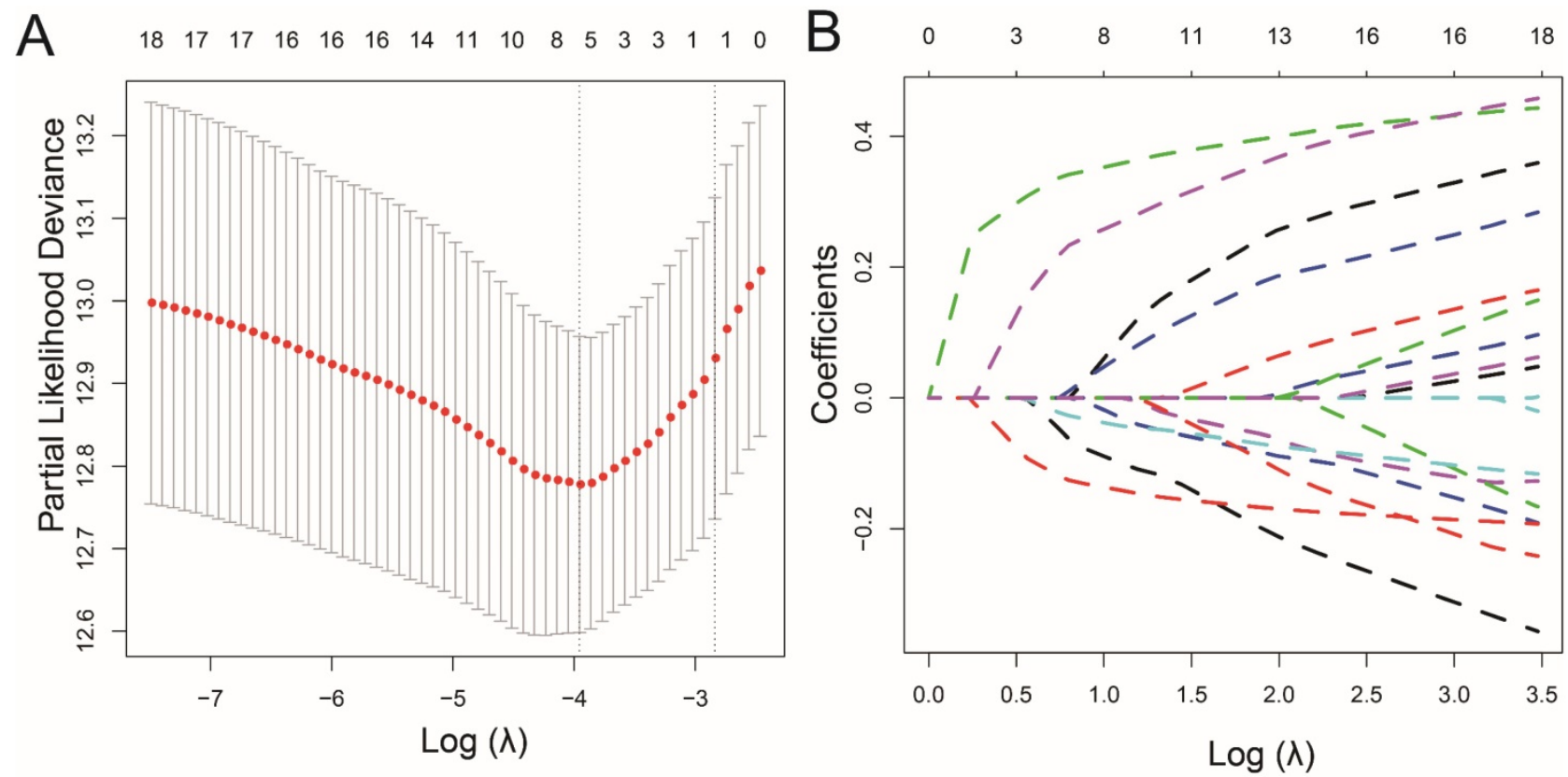

Figure 2. LASSO analysis to screen the candidates for prognostic signature. (A) The optimal tuning parameter (Lambda) in the LASSO model selected with the 10 -fold cross-validation and one standard error rule; (B) LASSO coefficient profiles of the 18 MMPs. 
prognosis with the HR of $2.54(\mathrm{P}=0.001$, Figure 5A), the AUC of PCa recurrence at 1-year is high to 0.976, 0.711 for 3-year, and 0.646 for 5-year (Figure 5D). As to GSE70769, including $46 \mathrm{MMPS}-\mathrm{H}$ patients and 46 MMPS-L patients, the RFS time in MMPS-L group is longer $(\mathrm{HR}=2.6,95 \% \mathrm{CI}=1.41-4.8, \mathrm{P}=0.002)$, and the prediction accuracy is reasonable (1-year AUC: 0.738; 3-year AUC $=0.760$, 5-year $\mathrm{AUC}=0.682$, Figure 5E). The MMPS-H group in MSKCC cohort also displayed an unfavorable prognosis, and the HR value of the risk score is 2.19 , with the $95 \%$ CI from 1.06 to 4.55
$(\mathrm{P}=0.035$, Figure $5 \mathrm{C})$, and the short and long RFS prediction value is high than 0.65 (1-year AUC: 0.793; 3 -year AUC $=0.681,5$-year AUC $=0.658$, Figure 5F). To sum up, the MMP-related predict signature is with a high accuracy to judge the PCa recurrence.

\section{The MMPS is an independent risk for the prognosis of $\mathrm{PCa}$ patients}

To evaluate the prognostic value of MMPS for $\mathrm{PCa}$ patients, we conducted the multivariate Cox analysis in each cohort with the clinical features
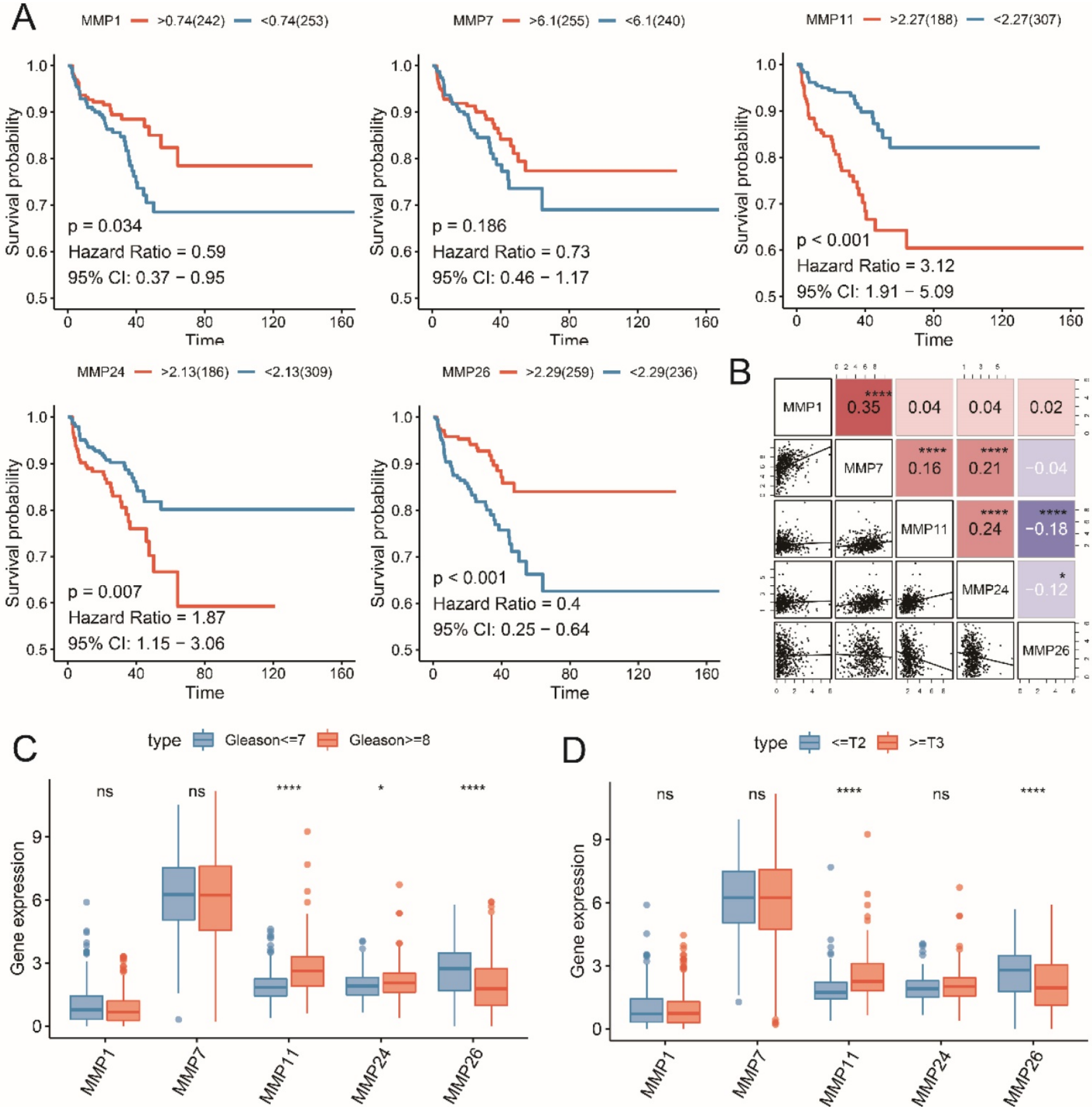

Figure 3. Five MMPs candidates associated with the RFS and pathological stage. (A) K-M plot showed the prognostic value of high and low level of five MMPs; (B) The correlation of mRNA expression of five MMPs; (C) The five MMPs expression in low and high Gleason score; (D) The five MMPs expression in early and advanced T stage. 
(Table 2). In TCGA-PRAD cohort, Gleason score (HR: 2.43, 95\%CI: 1.38-4.48, P=0.002), Stage (HR: 2.99, 95\% CI: 1.30-6.86, $\mathrm{P}=0.009)$, and MMPS risk (HR: 1.92, 95\% CI: $1.09-3.39, \mathrm{P}=0.025)$ were the independent risk for the RFS outcome. The 248 patients in the GSE116918 cohort also illustrated the independent predict value of the MMPS (HR: 2.55, 95\%CI: 1.05-6.19, $\mathrm{P}=0.039$ ). As to GSE70769 cohort, Stage (HR: 3.09, 95\% CI: 1.56-6.14, $\mathrm{P}=0.001$ ), and MMPS risk (HR: 2.35, 95\%CI: 1.22-4.51, $\mathrm{P}=0.010)$ acted as the independent risk for the RFS outcome. We failed to observe the independent predict value of MMPS (HR: 1.16, 95\% CI: 0.58-2.34, $\mathrm{P}=0.67$ ) after adjusting with the clinical features in MSKCC cohort.

\section{The potential MMPs driven mechanism to promote the progression of $\mathrm{PCa}$}

To explore the possible mechanism caused by MMPs in the tumorigenesis of $\mathrm{PCa}$, we firstly analyzed the DEGs in MMPS-H and MMP-L patients' groups. There are 394 up-regulated genes and 92 down-regulated genes with the cut-off fold change of 0.5 and P-value $<0.05$ (Figure 6A). COMP gene is the most elevated gene in MMPS-H group; with the help of K-M curve, we could see that the higher COMP, the higher risk of PCa recurrence (Figure 6B), and the association between COMP expression and risk score is high to 0.54 (Figure 6C). These results showed that the COMP is the potential MMPs driven downstream gene. We also use the Metascape to assess the gene enrichment of the upgrade of 394 genes. Not surprisingly, these genes are most enriched in GO:0030198: extracellular matrix organization, while the M18: Pid integrin1 pathway, M5901: HALLMARK G2M checkpoint and GO:0070848: response to growth factor were also illustrated, these pathways might be the target of PCa diagnosis and treatment.

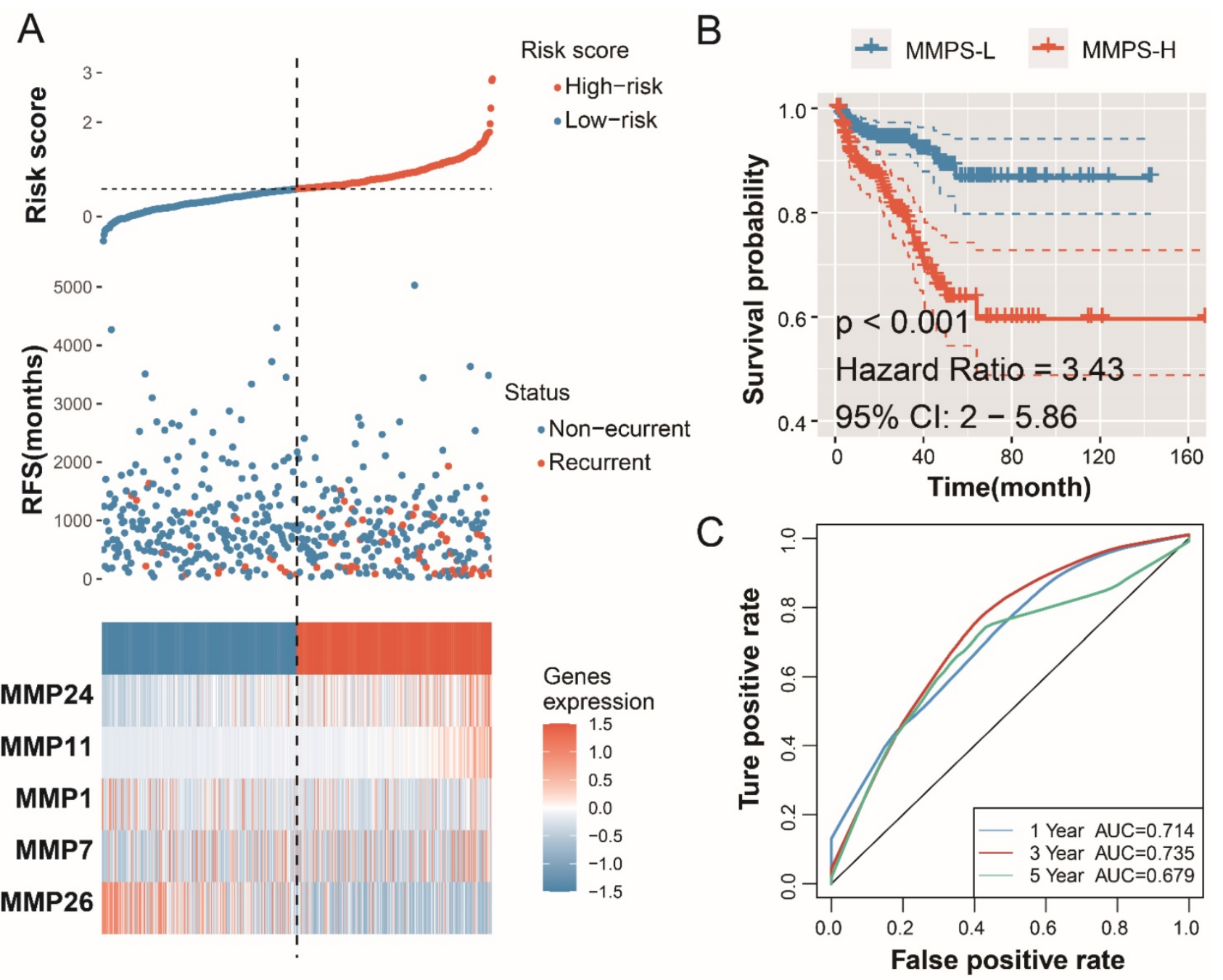

Figure 4. Establishment of the MMP-related prognostic model in training TCGA-PRAD cohort. (A) The risk score, recurrence status and five MMPs expression level; (B) K-M plot showed the RFS results of the MMPS-H (orange) and MMPS-L (blue) groups. (C) The 1-year, 3-year and 5-year ROC curves in the training group. 
A GSE116918 Cohort
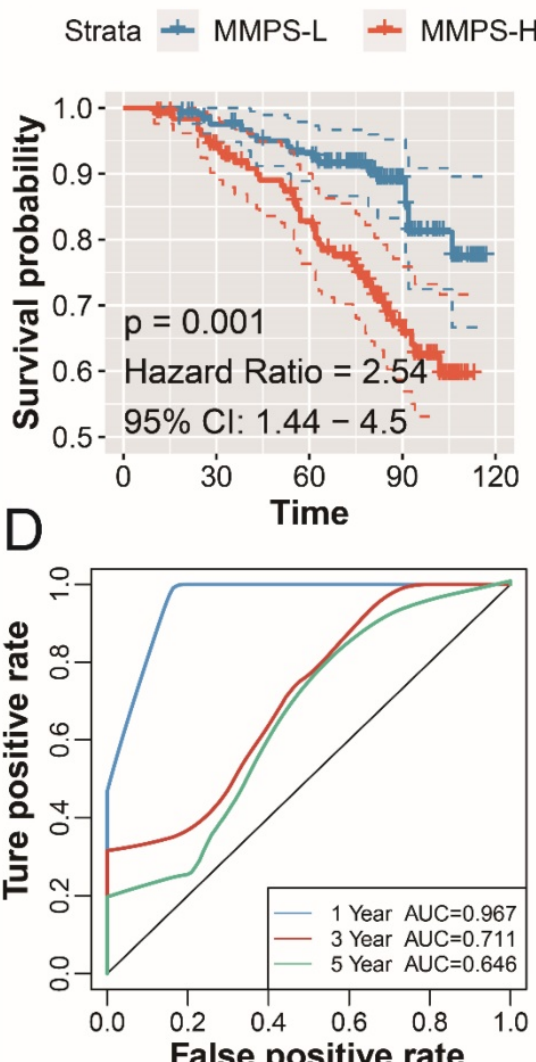

B

GSE70769 Cohort

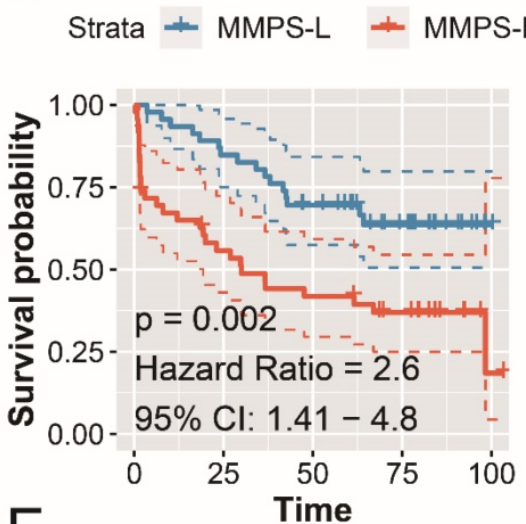

$\mathrm{E}$

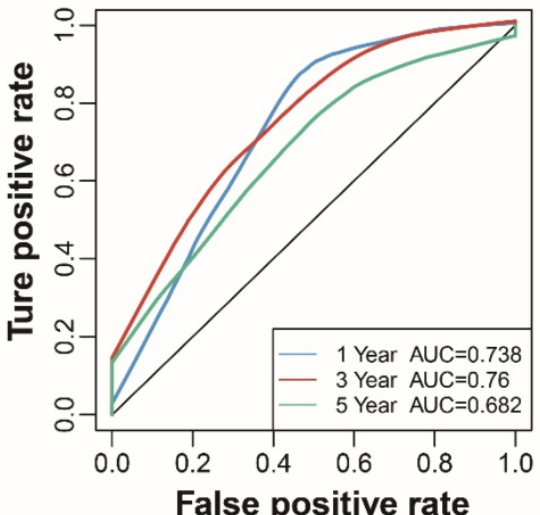

MSKCC Cohort

Strata $\neq$ MMPS-L $\perp$ MMPS-H

Figure 5. Validation of the MMP-related prognostic model in external cohorts. K-M plot showed the RFS results of the MMPS-H (orange) and MMPS-L (blue) groups in GSE1 16918 cohort (A), GSE70769 cohort (B) and MSKCC cohort (C); The 1-year, 3-year and 5-year ROC curves in GSE1 16918 cohort (D), GSE70769 cohort (E) and MSKCC cohort (F).

Table 2. Multivariate Cox analysis among MMPS signature and clinicopathological features.

\begin{tabular}{|c|c|c|c|}
\hline \multirow[t]{2}{*}{ Parameters } & \multicolumn{3}{|c|}{ Recurrence-free Survival } \\
\hline & HR & $95 \% \mathrm{CI}$ & P value \\
\hline \multicolumn{4}{|l|}{ TCGA-PRAD } \\
\hline Age (> 60 vs. $\leq 60)$ & 1.14 & $(0.70-1.85)$ & 0.595 \\
\hline Gleason score ( $\geq 8$ vs. $\leq 7$ ) & 2.43 & $(1.38-4.48)$ & $0.002^{*}$ \\
\hline Stage ( $\geq \mathrm{T} 3$ vs. $\leq \mathrm{T} 2)$ & 2.99 & $(1.30-6.86)$ & $0.009^{*}$ \\
\hline Risk (MMPS-H vs. MMPS-L) & 1.92 & $(1.09-3.39)$ & $0.025^{*}$ \\
\hline \multicolumn{4}{|l|}{ GSE116918 } \\
\hline Age (> 60 vs. $\leq 60)$ & 0.83 & $(0.38-1.8)$ & 0.634 \\
\hline Gleason score ( $\geq 8$ vs. $\leq 7$ ) & 0.53 & $(0.21-1.31)$ & 0.169 \\
\hline Stage $(\geq \mathrm{T} 3$ vs. $\leq \mathrm{T} 2)$ & 1.69 & $(0.93-3.05)$ & 0.084 \\
\hline Risk (MMPS-H vs. MMPS-L) & 2.55 & $(1.05-6.19)$ & $0.039^{*}$ \\
\hline \multicolumn{4}{|l|}{ GSE70769 } \\
\hline Gleason score ( $\geq 8$ vs. $\leq 7$ ) & 2.05 & $(0.99-4.22)$ & 0.05 \\
\hline Stage $(\geq \mathrm{T} 3$ vs. $\leq \mathrm{T} 2)$ & 3.09 & $(1.56-6.14)$ & $0.001^{*}$ \\
\hline Risk (MMPS-H vs. MMPS-L) & 2.35 & $(1.22-4.51)$ & $0.010^{*}$ \\
\hline \multicolumn{4}{|l|}{ MSKCC } \\
\hline Age $(>60$ vs. $\leq 60)$ & 0.80 & $(0.39-1.64)$ & 0.543 \\
\hline Gleason score ( $\geq 8$ vs. $\leq 7$ ) & 8.09 & $(3.79-17.24)$ & $<0.001^{*}$ \\
\hline Stage ( $\geq \mathrm{T} 3$ vs. $\leq \mathrm{T} 2)$ & 2.97 & $(1.36-6.47)$ & $0.006^{*}$ \\
\hline Risk (MMPS-H vs. MMPS-L) & 1.16 & $(0.58-2.34)$ & 0.67 \\
\hline
\end{tabular}

Immune infiltration of 22 type immunocytes

The results of the infiltration rate of 22 type immunocytes was generated by CIBERSORT. After comparing the infiltration of immunocytes of patients among MMPS-H and MMPS-L groups, we revealed that the plasma cells and resting mast cells decreased in MMPS-H group (all, $\mathrm{P}<0.05$ ), while activated CD4+ memory $\mathrm{T}$ cells, M1, M2 macrophages and resting dendritic cells increased in MMPS-H group (all, $\mathrm{P}<0.05$ ) (Figure 7A). We also found that the M2 macrophages are highly associated with the risk score generated by the MMP-related predict signature $(R=0.24$, Figure 7B). Meng et al. [26] recently reported that the M2 macrophage is a risk factor of $\mathrm{PCa}$ patients, Fakih et al. [27] reported a method to use the optimal cut-off to divide the enrolled patients to four groups by the scatter plot of two factors. Therefore, we use this method to separate the 496 TCGA-PRAD patients into four groups (Figure 7C). Interestingly, we found that the patients with low risk score all shown a better prognosis (Group I and III), no matter the infiltration of M2 macrophage is high or low, patients with the high MMPS risk score and low M2 macrophage showed the worst RFS (Figure 7D). These results showed that the MMPS score is an excellent signature to predict the prognosis. 

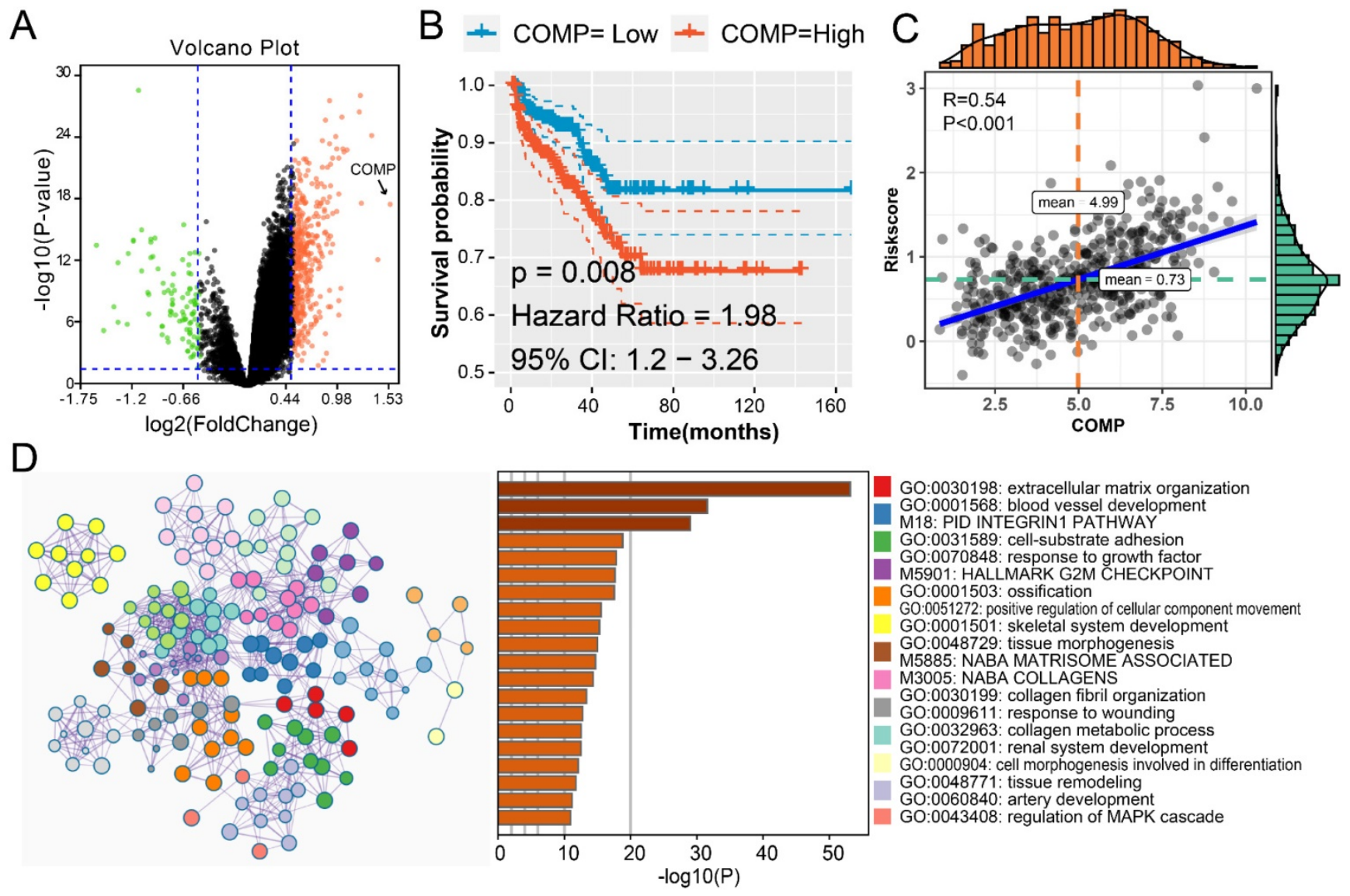

Figure 6. The MMPs driven mechanisms in MMPS-H group. (A) Volcano plot showed the DEGs among MMPS-H and MMPS-L groups; (B) K-M plot showed the RFS results of high and low mRNA level of COMP. (C) The correlation between COMP expression and MMPS risk score. (D) The pathway enrichment results of the 392 up-regulated genes in MMPS-H group.

\section{Discussion}

Cancer invasion through dense extracellular matrices (ECMs) is mediated by MMPs, which degrade the ECM thereby creating paths for migration $[28,29]$. Mounting evidence has revealed the function of MMPs in the past years and MMPs are the pivotal mediators for the microenvironment alteration determined tumorigenesis [30, 31]. The association between MMPs and PCa patients has also been widely studied. Białkowska et al. [32] reported that MMP7 rs11568818 polymorphism is correlated with the two-fold change of PCa risk, while MMP-1 rs1799750, MMP-2 rs243865, MMP13 rs2252070 not impact the risk. Ganguly et al. [33] found that Notch3 could promote the bone metastasis of PCa patients throng MMP3 mediated osteoblastic lesion formation. Kalantari et al. [34] revealed the bipartite function of MMP13 and TLR-9, patients with the high expression of MMP13 and TLR-9 showed an advanced stage of PCa. The status of CRPC and medicine resistance are the hot potatoes for the clinical treatment of $\mathrm{PCa}$ patients. Szarvas et al. [35] exposed higher pretreatment serum of MMP7 is the independent predictor of shorter cancer-specific survival and the resistance of docetaxel. Based on the above evidence, MMPs play an indispensable role in the initial alteration and development of PCa.

Due to the high recurrence rate and poor outcome of advanced PCa, and several researchers built the prognosis predict features to judge the outcome and provide more effeteness treatment for PCa patients. Toth et al. [36] generated a DNA methylation-based prognosis signature with the AUC of 0.95 in the training cohort, however, the AUC value in two external validation cohorts are only 0.771 and 0.687. Shao et al. [37] produced a seven long noncoding RNAs signature to predict the RFS of PCa, with the AUC value of 0.68 and C-index value of 0.63 , whereas this study lacks external validation. Jiang et al. [38] developed a 15-gene signature using Elastic-net analysis, the signature showed a predict AUC value of 0.766 at 11.5 months, 0.738 at 22.3 months, and 0.764 at 48.4 months. Therefore, it is meaningful to establish the prognosis predict signature to distinguish the low risk and high-risk PCa patients, as well as provide the appropriate treatment for them. 
A type
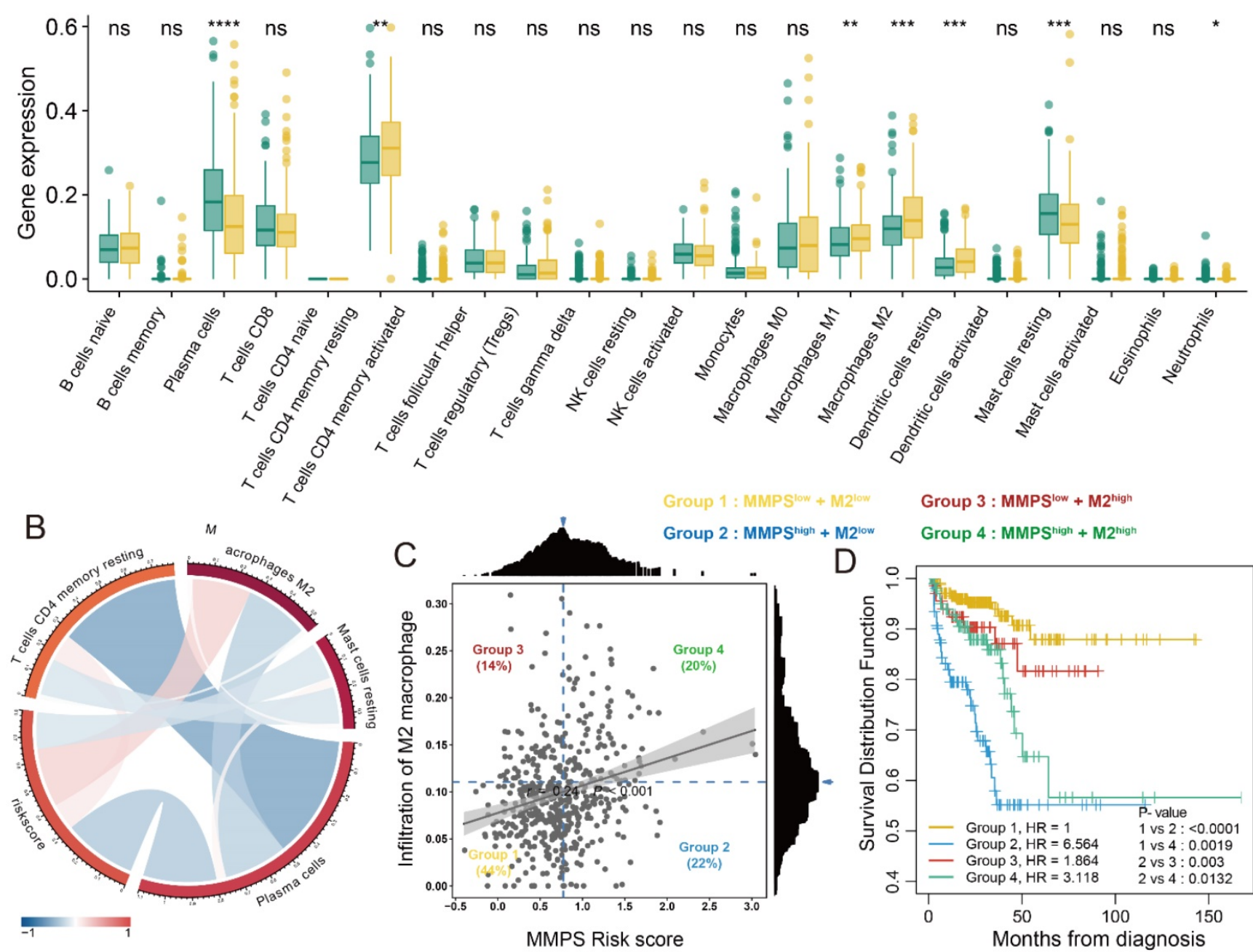

Figure 7. Immune infiltration of 22 immunocytes and association with prognosis in PCa patients. (A) Immune infiltration of 22 immunocytes in MMPS-L and MMPS-H groups; (B) The correlations between MMPS risk score and four immunocytes; (C) The optimal cut-off value of MMPS risk score and M2 macrophage infiltration to divide the enrolled patients to four groups; (D) K-M plot showed the different RFS of the four groups.

In the current study, we comprehensively assess the expression and prognostic value of $22 \mathrm{MMPs}$ in PCa patients. The mRNA level of MMPs in tumor and normal tissues is polarized, part of them increased in tumor tissues, part of them decreased in tumor tissues. What's more, about half of MMPs shown the negative relationship between the DNA methylation and mRNA expression, while genetic alteration is demonstrated no effect of mRNA level. Subsequently, the LASSO cox analysis was employed to dimensionality reduction and chose the MMPs to build the prognostic signature, MMP8, MMP21, MMP23B and MMP27 were excluded because of the lower expression of them. Finally, an MMP-related predict signature was obtained with the 1-year AUC of 0.714 in TCGA-PRAD training cohort and validated in three external cohorts with a high AUC value, including GSE116918, GSE70769 and MSKCC cohorts. What's more, after adjusting with the clinical features, we revealed that the MMPS signature is a robust independent predict toll for the RFS prognosis in $\mathrm{PCa}$ patients. The potential MMP-driven mechanisms also evaluated, and we reveled that Pid integrin1 pathway, G2M checkpoint and response to growth factor were the signaling pathways affected by MMPs. The positive associative between COMP and MMPS signature were also observed in this study. Liu et al. [39] reported that COMP is the biomarker for colon cancer and could promote the cell proliferation through Akt pathway. Stracke et al. [40] reported that MMP-19 may participate in the degradation of aggrecan and COMP in arthritic disease, whereas MMP-20 may primarily be involved in the turnover of these molecules during tooth development. Immunocyte infiltration was also concerned in this study, and found that the high risk score with the low infiltration of M2 macrophage shown the worst outcome in PCa patients. Based on the results 
generated from the current study, we could confirm the predict value of MMPS in PCa patients, in the future, if a patient diagnosed with the PCa and also obtained the high risk score of MMPS, we should take the active treatment to help him for the saving of the life.

\section{Conclusion}

MMPs involved and played an essential role in the tumorigenesis and biochemical recurrence in PCa patients. The MMPS signature could accurately predict the recurrence of PCa patients, and validated in several cohorts. The MMPs could affect the progression of PCa through Pid integrin1 pathway, G2M checkpoint and response to growth factor pathways.

\section{Acknowledgements}

The research was supported by the National Natural Science Foundation of China (No.81772708).

\section{Competing Interests}

The authors have declared that no competing interest exists.

\section{References}

1. Ferlay J, Soerjomataram I, Dikshit R, Eser S, Mathers C, Rebelo M, et al. Cancer incidence and mortality worldwide: sources, methods and major patterns in GLOBOCAN 2012. Int J Cancer. 2015; 136: E359-86.

2. Center MM, Jemal A, Lortet-Tieulent J, Ward E, Ferlay J, Brawley O, et al. International variation in prostate cancer incidence and mortality rates. Eur Urol. 2012; 61: 1079-92.

3. Bray F, Ferlay J, Soerjomataram I, Siegel RL, Torre LA, Jemal A. Global cancer statistics 2018: GLOBOCAN estimates of incidence and mortality worldwide for 36 cancers in 185 countries. CA Cancer J Clin. 2018; 68: 394-424.

4. Halabi S, Lin CY, Kelly WK, Fizazi KS, Moul JW, Kaplan EB, et al. Updated prognostic model for predicting overall survival in first-line chemotherapy for patients with metastatic castration-resistant prostate cancer. J Clin Oncol. 2014; 32: 671-7.

5. Kirby M, Hirst C, Crawford ED. Characterising the castration-resistant prostate cancer population: a systematic review. Int J Clin Pract. 2011; 65: 1180-92.

6. Howard N, Clementino M, Kim D, Wang L, Verma A, Shi X, et al. New developments in mechanisms of prostate cancer progression. Semin Cancer Biol. 2018.

7. Virgo KS, Basch E, Loblaw DA, Oliver TK, Rumble RB, Carducci MA, et al. Second-Line Hormonal Therapy for Men With Chemotherapy-Naive, Castration-Resistant Prostate Cancer: American Society of Clinical Oncology Provisional Clinical Opinion. J Clin Oncol. 2017; 35: 1952-64.

8. Nagase H, Visse R, Murphy G. Structure and function of matrix metalloproteinases and TIMPs. Cardiovasc Res. 2006; 69: 562-73.

9. Marchant DJ, Bellac CL, Moraes TJ, Wadsworth SJ, Dufour A, Butler GS, et al. A new transcriptional role for matrix metalloproteinase-12 in antiviral immunity. Nat Med. 2014; 20: 493-502.

10. Wang X, Khalil RA. Matrix Metalloproteinases, Vascular Remodeling, and Vascular Disease. Adv Pharmacol. 2018; 81: 241-330.

11. Murphy G, Nagase H. Progress in matrix metalloproteinase research. Mol Aspects Med. 2008; 29: 290-308.

12. Quintero-Fabian S, Arreola R, Becerril-Villanueva E, Torres-Romero JC, Arana-Argaez V, Lara-Riegos J, et al. Role of Matrix Metalloproteinases in Angiogenesis and Cancer. Front Oncol. 2019; 9: 1370.

13. Bulboaca AE, Boarescu PM, Porfire AS, Dogaru G, Barbalata C, Valeanu $\mathrm{M}$, et al. The Effect of Nano-Epigallocatechin-Gallate on Oxidative Stress and Matrix Metalloproteinases in Experimental Diabetes Mellitus. Antioxidants (Basel). 2020; 9 .
14. Niu Q, Xing F, Gu HW, Bai L, Zhang J, Yuan JJ, et al. Upregulation of Myeloid Zinc Finger 1 in Dorsal Root Ganglion via Regulating Matrix Metalloproteinase-2/9 and Voltage-gated Potassium 1.2 Expression Contributes to Complete Freund's Adjuvant-induced Inflammatory Pain. Neuroscience. 2020; 432: 174-87.

15. Provenzano M, Andreucci M, Garofalo C, Faga T, Michael A, Ielapi N, et al. The Association of Matrix Metalloproteinases with Chronic Kidney Disease and Peripheral Vascular Disease: A Light at the End of the Tunnel? Biomolecules. 2020; 10.

16. Rivera S, Garcia-Gonzalez L, Khrestchatisky M, Baranger K. Metalloproteinases and their tissue inhibitors in Alzheimer's disease and other neurodegenerative disorders. Cell Mol Life Sci. 2019; 76: 3167-91.

17. Gobin E, Bagwell K, Wagner J, Mysona D, Sandirasegarane S, Smith N, et al. A pan-cancer perspective of matrix metalloproteases (MMP) gene expression profile and their diagnostic/prognostic potential. BMC Cancer. 2019; 19: 581.

18. Qi S, Perrino S, Miao X, Lamarche-Vane N, Brodt P. The chemokine CCL7 regulates invadopodia maturation and MMP-9 mediated collagen degradation in liver-metastatic carcinoma cells. Cancer Lett. 2020.

19. Furuya H, Pagano I, Chee K, Kobayashi T, Wong RS, Lee R, et al. Comparison of Commercial ELISA Kits, a Prototype Multiplex Electrochemoluminescent Assay, and a Multiplex Bead-Based Immunoassay for Detecting a Urine-Based Bladder-Cancer-Associated Diagnostic Signature. Diagnostics (Basel). 2019; 9.

20. Singh R, Dagar P, Pal S, Basu B, Shankar BS. Significant alterations of the novel 15 gene signature identified from macrophage-tumor interactions in breast cancer. Biochim Biophys Acta Gen Subj. 2018; 1862: 669-83.

21. Cerami E, Gao J, Dogrusoz U, Gross BE, Sumer SO, Aksoy BA, et al. The cBio cancer genomics portal: an open platform for exploring multidimensional cancer genomics data. Cancer Discov. 2012; 2: 401-4.

22. Gao J, Aksoy BA, Dogrusoz U, Dresdner G, Gross B, Sumer SO, et al. Integrative analysis of complex cancer genomics and clinical profiles using the cBioPortal. Sci Signal. 2013; 6: pl1.

23. Zhou Y, Zhou B, Pache L, Chang M, Khodabakhshi AH, Tanaseichuk O, et al. Metascape provides a biologist-oriented resource for the analysis of systems-level datasets. Nat Commun. 2019; 10: 1523.

24. Chen B, Khodadoust MS, Liu CL, Newman AM, Alizadeh AA. Profiling Tumor Infiltrating Immune Cells with CIBERSORT. Methods Mol Biol. 2018; 1711: 243-59.

25. Li T, Fan J, Wang B, Traugh N, Chen Q, Liu JS, et al. TIMER: A Web Server for Comprehensive Analysis of Tumor-Infiltrating Immune Cells. Cancer Res. 2017; 77: e108-e10.

26. Meng J, Liu Y, Guan S, Fan S, Zhou J, Zhang M, et al. The establishment of immune infiltration based novel recurrence predicting nomogram in prostate cancer. Cancer Med. 2019; 8: 5202-13.

27. Fakih M, Ouyang C, Wang C, Tu TY, Gozo MC, Cho M, et al. Immune overdrive signature in colorectal tumor subset predicts poor clinical outcome. J Clin Invest. 2019; 129: 4464-76.

28. Bonnans C, Chou J, Werb Z. Remodelling the extracellular matrix in development and disease. Nat Rev Mol Cell Biol. 2014; 15: 786-801.

29. Kumar S, Kapoor A, Desai S, Inamdar MM, Sen S. Proteolytic and non-proteolytic regulation of collective cell invasion: tuning by ECM density and organization. Sci Rep. 2016; 6: 19905.

30. Kessenbrock K, Plaks V, Werb Z. Matrix metalloproteinases: regulators of the tumor microenvironment. Cell. 2010; 141: 52-67.

31. Noel A, Gutierrez-Fernandez A, Sounni NE, Behrendt N, Maquoi E, Lund IK, et al. New and paradoxical roles of matrix metalloproteinases in the tumor microenvironment. Front Pharmacol. 2012; 3: 140.

32. Bialkowska K, Marciniak W, Muszynska M, Baszuk P, Gupta S, Jaworska-Bieniek K, et al. Association of zinc level and polymorphism in MMP-7 gene with prostate cancer in Polish population. PLoS One. 2018; 13: $\mathrm{e} 0201065$.

33. Ganguly SS, Hostetter G, Tang L, Frank SB, Saboda K, Mehra R, et al. Notch3 promotes prostate cancer-induced bone lesion development via MMP-3. Oncogene. 2020; 39: 204-18.

34. Kalantari E, Abolhasani M, Roudi R, Farajollahi MM, Farhad S, Madjd Z, et al. Co-expression of TLR-9 and MMP-13 is associated with the degree of tumour differentiation in prostate cancer. Int J Exp Pathol. 2019; 100: 123-32.

35. Szarvas T, Sevcenco S, Modos O, Keresztes D, Nyirady P, Csizmarik A, et al. Matrix metalloproteinase 7, soluble Fas and Fas ligand serum levels for predicting docetaxel resistance and survival in castration-resistant prostate cancer. BJU Int. 2018; 122: 695-704.

36. Toth R, Schiffmann H, Hube-Magg C, Buscheck F, Hoflmayer D, Weidemann $\mathrm{S}$, et al. Random forest-based modelling to detect biomarkers for prostate cancer progression. Clin Epigenetics. 2019; 11: 148. 
37. Shao N, Zhu Y, Wan FN, Ye DW. Identification of seven long noncoding RNAs signature for prediction of biochemical recurrence in prostate cancer. Asian J Androl. 2019; 21: 618-22.

38. Jiang Y, Mei W, Gu Y, Lin X, He L, Zeng H, et al. Construction of a set of novel and robust gene expression signatures predicting prostate cancer recurrence. Mol Oncol. 2018; 12: 1559-78.

39. Liu TT, Liu XS, Zhang M, Liu XN, Zhu FX, Zhu FM, et al. Cartilage oligomeric matrix protein is a prognostic factor and biomarker of colon cancer and promotes cell proliferation by activating the Akt pathway. J Cancer Res Clin Oncol. 2018; 144: 1049-63.

40. Stracke JO, Fosang AJ, Last K, Mercuri FA, Pendas AM, Llano E, et al. Matrix metalloproteinases 19 and 20 cleave aggrecan and cartilage oligomeric matrix protein (COMP). FEBS Lett. 2000; 478: 52-6. 